\title{
Multiple multilayer stents for thoracoabdominal aortic aneurysm: a possible new tool for aortic endovascular surgery
}

This article was published in the following Dove Press journal:

International Journal of General Medicine

23 July 2012

Number of times this article has been viewed

\author{
Valerio Stefano Tolva ${ }^{1,3}$ \\ Paolo Guy Bianchi' \\ Lea Valeria Cireni' \\ Alma Lombardo' \\ Guido Carlo Keller' \\ Gianfranco Parati ${ }^{2,3}$ \\ Renato Maria Casana' \\ 'Surgical Department Istituto \\ Auxologico Italiano IRCCS, Milan, \\ Italy; ${ }^{2}$ Department of Cardiology, \\ Istituto Auxologico Italiano, Università \\ degli Studi di Milano-Bicocca, Bicocca, \\ Milan, Italy; ${ }^{3}$ Department of Internal \\ Medicine, San Gerardo Hospital, \\ Università degli Studi di Milano- \\ Bicocca, Bicocca, Milan, Italy
}

Purpose: Endovascular surgery data are confirming the paramount role of modern endovascular tools for a safe and sure exclusion of thoracoabdominal lesions.

Case report: A 57-year-old female presented with severe comorbidity affected by a $58 \mathrm{~mm}$ thoracoabdominal aortic aneurysm (TAAA). After patient-informed consent and local Ethical Committee and Italian Public Health Ministry authorization, three multilayer stents were implanted in the thoracoabdominal aortic tract, obtaining at a 20-month computed tomography scan follow up, a complete exclusion of the TAAA, with normal patency of visceral vessels.

Conclusion: Multilayer stents can be used in thoracoabdominal aortic aneurysm, with positive results.

Keywords: chimney technique, renal artery stenosis, thoracoabdominal aneurysm, vascular complication

\section{Introduction}

The first target of endovascular aneurysm repair (EVAR) for all aortic lesions is to seal the graft to safe aortic tissue. Endoleaks and endotension remain the primary limitation of endovascular aortic repair with endografts. ${ }^{1}$ Midterm secondary intervention rate for endovascular aortic repairs, based on the largest and most recent datasets, is between $7 \%$ and $12 \%$ for both EVAR and thoracic EVAR, with most reinterventions being done for endoleaks. ${ }^{1-3}$ Secondary intervention is mandatory when an endoleak has resulted in serious complications, including colonic ischemia and paraparesis. ${ }^{4,5}$ Techniques such as chimney-stent (Ch-EVAR), ${ }^{6}$ graft fenestration (F-EVAR), ${ }^{7}$ or debranching $^{8}$ help in shifting the endograft landing zone to a safer proximal position, but the complete endovascular approach is a significant risk factor for kidney injury, chronic renal failure, and bowel complications..$^{6-8}$

\section{Case report}

A 57-year-old obese female (body mass index 57.7), affected by chronic obstructive pulmonary disease, mild arterial hypertension, moderate renal failure (plasmatic creatinine $1.9 \mathrm{mg} / \mathrm{dL}$, estimated glomerular filtration rate 58.8, calculated with the Chronic Kidney Disease Epidemiology Collaboration formula) with a complete occlusion of the right renal artery and a left renal artery severe stenosis (Figure 2) presented with a $58 \mathrm{~mm}$ thoracoabdominal aortic aneurysm (TAAA) (Figures 1 and 3). The left iliac artery (Figure 3) was occluded, and the patient suffered from a left leg disabling intermittent claudication. The right common femoral artery had a diameter of $25 \mathrm{~mm}$. Preoperative clinical and instrumental investigations were performed.
Correspondence: Valerio Stefano Tolva Istituto Auxologico Italiano IRCCS, Via P Lombardo 22, 20135 Milan, Italy Tel +390289421375

Email v.tolva@auxologico.it 


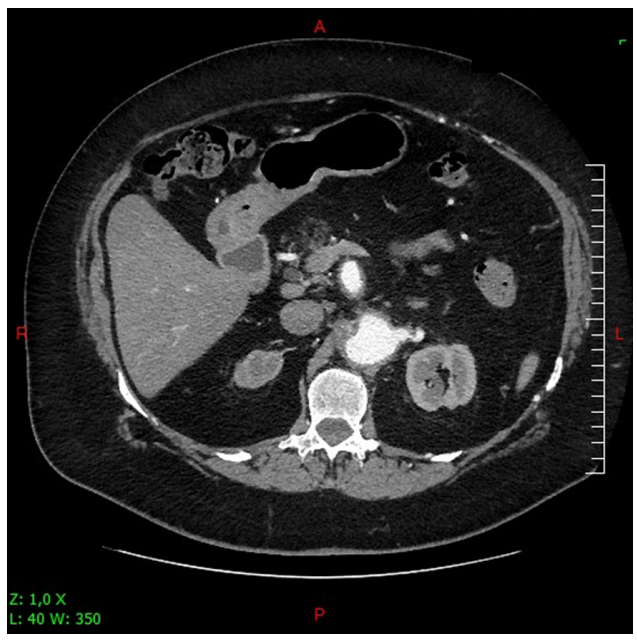

Figure I Computed tomography scan: left renal artery stenotic ostium in aneurismal aortic tract.

Ankle brachial index was 0.90 on the right leg and 0.40 on the left leg. Carotid Doppler ultrasound showed a mild bilateral internal carotid stenosis, and dobutamine-echocardiography was negative for cardiac-induced ischemia. After patient-informed consent and hospital ethical committee and Italian Public Health Ministry authorization, an endograft procedure was performed using Cardiatis ${ }^{\circledR}$ (Crossmed, Isnes, Belgium) multilayer stents in the aneurysmatic thoracoabdominal aorta. Stent graft sizing and deployment strategy was planned using tomographic aortography (CTA) images reconstructed on a three-dimensional workstation (Sovamed Gmbh, Koblenz, Germany). Proximal and distal neck diameter were 30 and $18 \mathrm{~mm}$ respectively. Stent oversizing was about $20 \%$ (proximal $36 \mathrm{~mm}$; distal $22 \mathrm{~mm}$ ). A multiple stenting (three grafts) with a $5 \mathrm{~cm}$ overlap, using telescopic

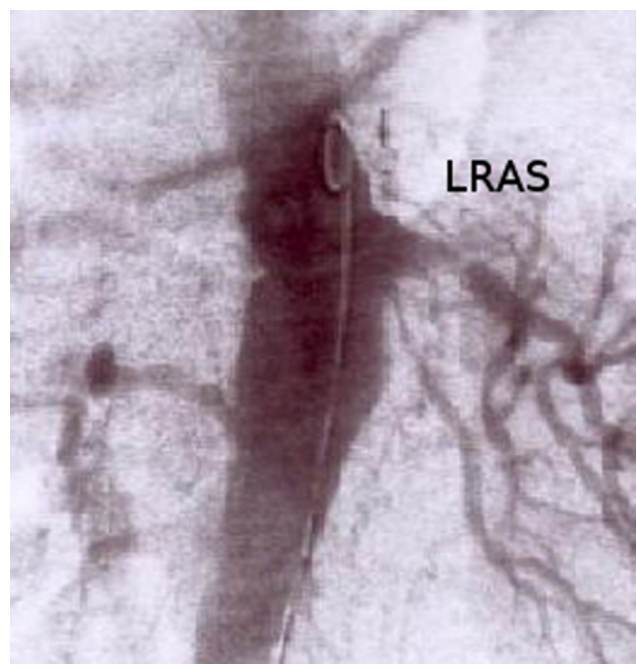

Figure 2 Intraoperative angiogram: left renal artery stenosis.

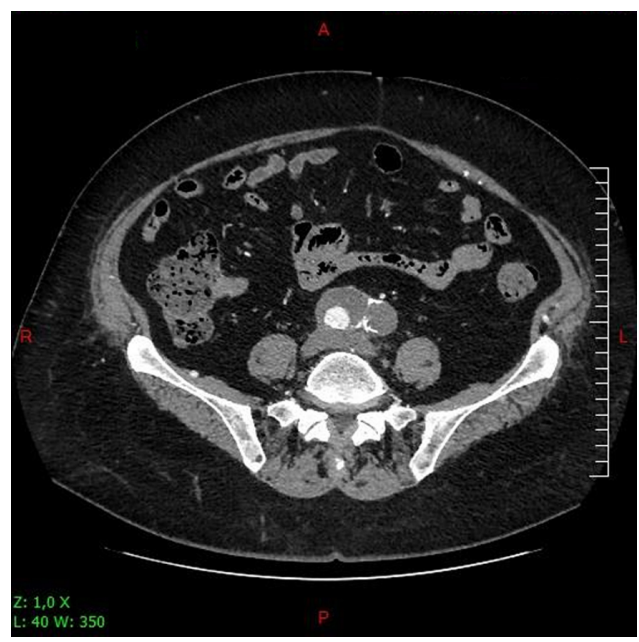

Figure 3 Computed tomography scan: left common iliac artery occlusion and right iliac artery aneurysm.

deployment, was planned. The procedure was performed by vascular surgeons in an operating room equipped with a C-Arm brilliance intensifier-General Electric OEC9800 (GE Healthcare, USA). The patient underwent subarachnoid anesthesia. Left renal stenting was primarily executed using a $5 \mathrm{~mm} \times 19 \mathrm{~mm}$ Herculink stent (Abbott Italia, Latina, Italy). Graft deployment started from the right common iliac artery using a 20-French introducer inserted into the aneurismal right femoral artery (RFA). Final angiograms showed normal patency of TAAA and visceral vessels (Figure 5).

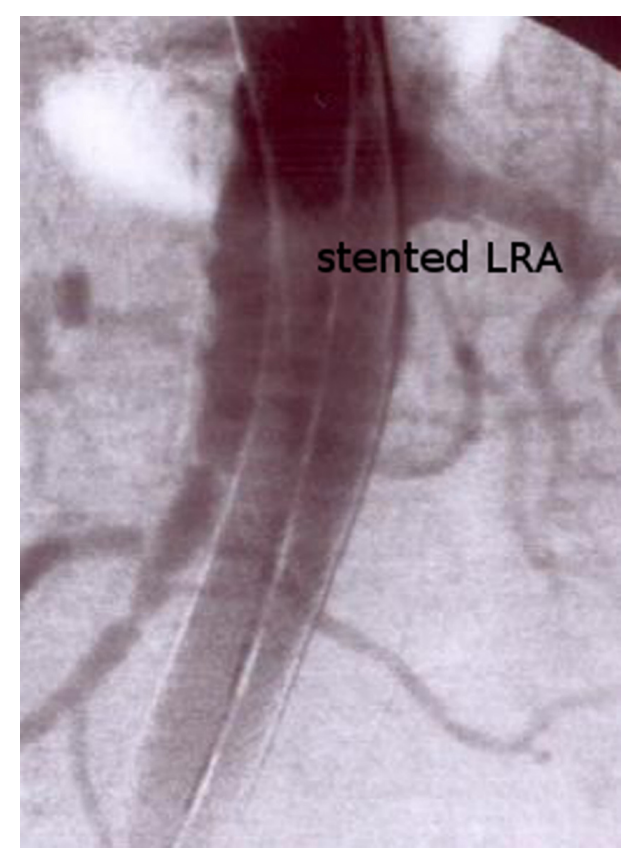

Figure 4 Intraoperative angiogram: good patency of $\mathrm{CT}$, superior mesenteric artery, and left renal artery.

Abbreviation: CT, computed tomography. 


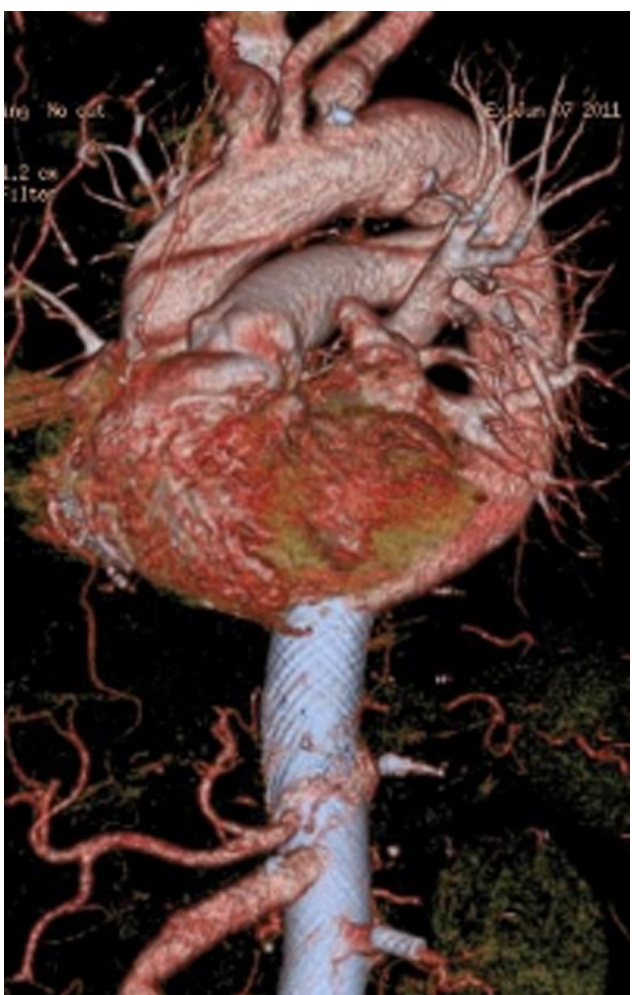

Figure 5 Computed tomography scan: treated thoracoabdominal aortic aneurysm.

Finally, after removing the introducer, the RFA was replaced with a $10 \mathrm{~mm}$ Dacron graft (MAQUET GmbH and Co, Rastatt, Germany), and a right to left femoro-femoral bypass was performed (10 mm Dacron graft; MAQUET GmbH and Co). Postoperative course was uneventful and postoperative day 10 CTA showed normal patency of the visceral vessels. The patient was dismissed on postoperative day 12, with $75 \mathrm{mg}$ of clopidogrel and $100 \mathrm{mg}$ aspirin daily oral intake. After 20 months, CTA showed good patency of the visceral vessels with complete TAAA exclusion (Figures 5 and 6 ).

\section{Discussion}

Treatment of pararenal and thoracoabdominal aortic aneurysms still represent a high risk surgical and endovascular procedure. When open surgery is considered, even in experienced centers, perioperative mortality rates range from $3 \%$ to $19 \%$. New technologies have expanded the applicability of endovascular aneurysm treatment to cases with anatomical adverse conditions. But as the indications for mini-invasive techniques have improved, complications and technical failure rates began to rise. ${ }^{1,8-10}$ Furthermore, the development of endograft technology and the evolution of branched and fenestrated grafts have necessitated the development of revised reporting standards for EVAR. ${ }^{11}$ During the setting

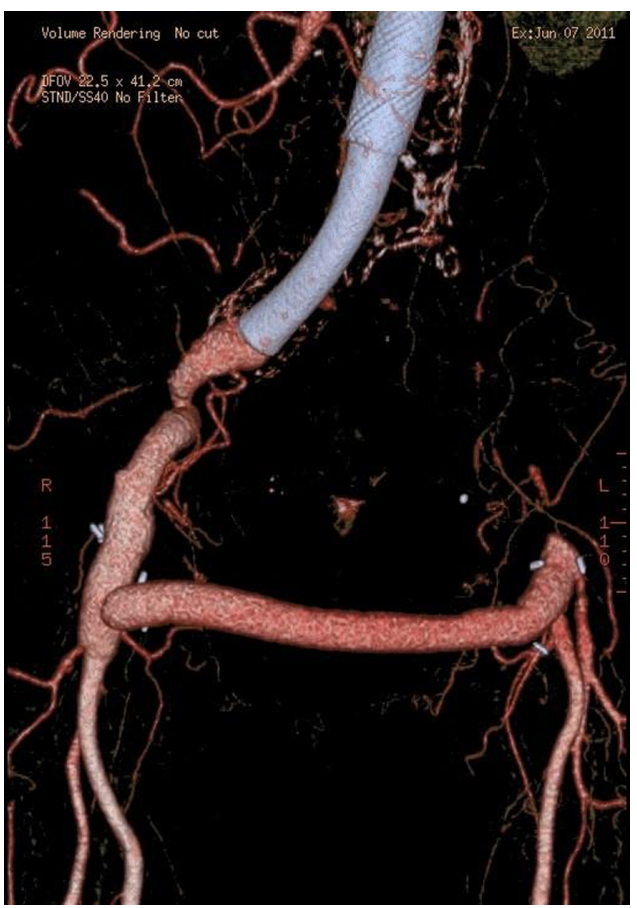

Figure 6 Computed tomography scan: treated thoracoabdominal aortic aneurysm and right to left femoro-femoral bypass.

and tailoring of the treatment for this patient, the multiple preoperative morbidities indicated that an endovascular technique was the first advisable approach. The initial aim was to protect the left kidney and thereafter exclude the TAAA. Aneurysm anatomy and proximal aortic neck was unsuitable for standard abdominal aortic endograft. The debranching technique for the abdominal open surgery time was excluded. Considering the computed tomography (CT) scan, a normal aortic diameter with an eccentric posterior thrombotic apposition (Figure 1) was noted on the superior mesenteric artery (SMA). Even if it is reported graft, fixation on thrombus may cause type I endoleak and so we preferred to deploy the graft in a safe landing zone. The maximum number of vessels that can be safely chimney-stented has never been systematically studied and remains unknown, but two chimneys seems to be the number for a safe procedure. ${ }^{9}$ If Ch-EVAR was used for this patient, the CT, SMA, and left renal artery would have had to be chimney-stented, but the authors considered Ch-EVAR too dangerous for renal and visceral complications. Evaluating F-EVAR, we faced a $15 \%$ of secondary intervention rate within the first year. Reintervention was for endoleak in $48 \%$ of cases; in the remaining $52 \%$, a significant amount was for mesenteric ischemia. ${ }^{12}$ Recently, Manning et al ${ }^{13}$ alerted about the risk in using triple fenestration grafting. The authors underline that at present the procedure is technically more demanding 
and associated with increased risk compared with double or single fenestrations. ${ }^{13}$ We decided for multilayer stent aortic grafting, after previously obtaining good results in peripheral artery aneurysm treatment. The multilayer stents (Cardiatis CrossMed, Isnes, Belgium) provides a new approach to the treatment of TAAA. Three-dimensional structure stents are made up of several layers braided together, creating a configuration of multiple interlocked layers. Experimental assessment of multilayer stents confirmed the optimal flow modulation through the layers was reached, with a $65 \%$ mean porosity. ${ }^{14,15}$ Secondary to this porosity level, when bridging an aneurysm or false dissection lumen, the stent laminates the blood flow by provoking a pressure drop. The flow velocity outside the multilayer stent is then reduced up to $90 \%$, creating an organized thrombus while preserving the laminar flow in the collateral arteries. Chocron et $\mathrm{al}^{14}$ reported good outcomes with multilayer stents in a type B residual dissection, obtaining a complete exclusion of the false lumen and patency of the visceral vessels.

\section{Conclusion}

The authors' clinical experience with multilayer stents has shown that they preserve the flow in the collateral arteries and exclude the aneurysm. The simplicity on releasing the devices and the absence of secondary procedures like Ch-EVAR or F-EVAR convinced the authors to perform this particular procedure for this patient. As a single experience, there are no "instructions for use," but a multilayer-stent graft could be considered an effective and satisfactory element in the endophile surgeon's toolbox.

\section{Disclosure}

The authors report no conflicts of interest in this work.

\section{References}

1. Gleason TG. Endoleaks after endovascular aortic stent-grafting: impact, diagnosis and management. Semin Thorac Cardiovasc Surg. 2009;21:363-372.
2. Espinosa G, Ribeiro Alves M, Ferreira Caramalho M, et al. 10-year single-center prospective study of endovascular abdominal aortic aneurysm repair with the talent stent graft. $J$ Endovasc Ther. 2009;16:125-135.

3. Morales JP, Greenberg RK, Lu Q, et al. Endoleaks following endovascular repair of thoracic aortic aneurysm: etiology and outcomes. $J$ Endovasc Ther. 2008;15:631-638.

4. Gambaro E, Abou-Zamzam AM Jr, Teruya TH, et al. Ischemic colitis following translumbar thrombin inijection for treatment of endoleak. Ann Vasc Surg. 2004;18:74-78.

5. Nevala T, Biancari F, Manninen H, et al. Type II endoleak after endovascular repair of abdominal aortic aneurysm: effectiveness of embolization. Cardiovasc Intervent Radiol. 2010;33:278-284.

6. Greenberg RK, Clair RD, Srivastava S, et al. Should patients with challenging anatomy be offered endovascular aneurysm repair? J Vasc Surg. 2003;38:990-996.

7. Verhoeven EL, Vourliotakis G, Bos WT, et al. Fenestrated stent grafting for short-neck and juxtarenal abdominal aortic aneurysm: an 8-year single-center experience. Eur J Vasc Endovasc Surg. 2010;39: $529-536$.

8. Hughes GC, McCann RL. Hybryd thoracoabdominal aortic aneurysm repair: concomitant visceral revascularization and endovascular aneurysm exclusion. Seminar Thorac Cardiovasc Surg. 2009;21:355-362.

9. Bruen KJ, Feezor RJ, Daniels MJ, Beck AW, Lee WA. Endovascular chimney technique versus open repair of iuxtarenal and suprarenal aneursyms. J Vasc Surg. 2011;53:895-904.

10. Sultan S, Hynes N. Clinical efficacy and cost per quality-adjusted life years of pararenal endovascular aortic aneurysm repair compared with open surgical repair. J Endovasc Ther. 2011;18:181-196.

11. Chaikof EL, Blankesteijn JD, Harris PL, et al. Reporting standards for endovascular aortic aneurysm repair. J Vasc Surg. 2002;35: 1048-1060.

12. Nordon IM, Hinchliffe RJ, Holt PJ, et al. Modern treatment of the juxtarenal abdominal aortic aneurysms with fenestrated endografting and open repair - a systematic review. Eur J Endovasc Surg. 2009;38:35-41.

13. Manning BJ, Obiekezie A, Richards T, Ivancev K, Harris PL. Early outcome following endovascular repair of pararenal repair of pararenal aortic aneurysms: triple- versus double- or single-fenestrated stentgrafts. J Endovasc Ther. 2011;18:98-105.

14. Chocron S, Vaislic C, Kaili D, Bonneville JF. Multilayer stents in the treatment of thoraco-abdominal residual type B dissection. Interact Cardiovasc Thorac Surg. 2011;12:1057-1059.

15. Balderi A, Antonietti A, Pedrazzini F, et al. Treatment of hepatic artery aneurysm by endovascular exclusion using the multilayer cardiatis stent. Cardiovasc Intervent Radiol. 2010;33:1282-1286.
International Journal of General Medicine

\section{Publish your work in this journal}

The International Journal of General Medicine is an international, peer-reviewed open-access journal that focuses on general and internal medicine, pathogenesis, epidemiology, diagnosis, monitoring and treatment protocols. The journal is characterized by the rapid reporting of reviews, original research and clinical studies across all disease areas.

\section{Dovepress}

A key focus is the elucidation of disease processes and management protocols resulting in improved outcomes for the patient.The manuscript management system is completely online and includes a very quick and fair peer-review system. Visit http://www.dovepress.com/ testimonials.php to read real quotes from published authors. 